\title{
Bioinformatic analysis of endothelial progenitor cells exposed to folic acid in type 1 diabetes mellitus
}

\author{
D.N. Fang ${ }^{1}$, X.D. He ${ }^{2}$, X.H. Li ${ }^{1}$, H. Jia ${ }^{3}$, P.Y. $\mathrm{Li}^{4}$, Q. $\mathrm{Lu}^{5}$, Z. Quan ${ }^{6}$ and \\ Q.L. Wang ${ }^{7}$ \\ ${ }^{1}$ Department of Gastroenterology, Shanghai Eighth People's Hospital, \\ Shanghai, China \\ ${ }^{2}$ Department of Endocrinology the First Affiliated Hospital of Xinjiang Medical \\ University, Xinjiang, China \\ ${ }^{3}$ Department of Emergency Medicine, \\ The Fourth Affiliated Hospital of China Medical University, Shenyang, China \\ ${ }^{4}$ Department of Anatomy and Embryology, \\ Shanghai Medical College of Fudan University, Shanghai, China \\ ${ }^{5}$ Department of Obstetrics and Gynecology, Central Hospital, \\ Fengxian District, Shanghai, China \\ ${ }^{6}$ Department of Neurosurgery, Central Hospital, Fengxian District, \\ Shanghai, China \\ ${ }^{7}$ Department of Histology and Embryology, School of Basic Medical, \\ Shanghai University of Traditional Chinese Medicine, Shanghai, China \\ Corresponding authors: Z. Quan / Q.L. Wang \\ E-mail: zhequanzq@hotmail.com / wlei810@gmail.com
}

Genet. Mol. Res. 13 (1): 1-10 (2014)

Received April 31, 2013

Accepted October 4, 2013

Published January 3, 2014

DOI http://dx.doi.org/10.4238/2014.January.3.1

ABSTRACT. We investigated the effects of type 1 diabetes mellitus (T1DM) on endothelial progenitor cells (EPCs) at the molecular level and assessed the therapeutic potential of folic acid (FA) in DM. We downloaded the gene expression profile of the EPCs from T1DM patients before and after treatment with FA and from healthy controls. We identified the 
differentially expressed genes (DEGs) in the EPCs from T1DM patients before and after a four-week period of FA treatment and compared them with those obtained from the healthy subjects by using limma package in $\mathrm{R}$ language. Then, functional annotation of the DEGs was performed using the online tool Database for Annotation, Visualization and Integrated Discovery (DAVID) based on the Kyoto Encyclopedia of Genes and Genomes database. The expression of 696 genes was altered in the EPCs from T1DM patients compared to those from the healthy controls. These genes were mainly involved in the pathways associated with immune response. FA can normalize majority of the altered gene expression profiles of EPCs from T1DM patients to resemble those of healthy subjects, albeit with some side effects. FA can be a potential therapeutic agent for the treatment of T1DM. However, focused efforts are required to ensure that the dose of FA falls within the permissible pharmacological range.

Key words: Type 1 diabetes mellitus; Folic acid; Immune response; Endothelial progenitor cells; Differentially expressed genes

\section{INTRODUCTION}

Type 1 diabetes mellitus (T1DM) is a form of diabetes mellitus that results from autoimmunity-induced destruction of insulin-producing beta cells of the pancreas (Atkinson and Maclaren, 1994; Schranz and Lernmark, 1998). It generally develops in the young, and accounts for approximately $5-10 \%$ of the diabetic population worldwide (Daneman, 2006). Individuals with diabetes mellitus are at high risk for both microvascular and macrovascular complications, and are associated with endothelial dysfunction, premature atherosclerosis, and reduced ability for angiogenesis in ischemic conditions (Williams et al., 1996; Sheetz and King, 2002; Nathan et al., 2003).

T1DM is a multifactorial process, and both genetic and environmental factors are involved in its initiation and progression. Many studies have shown that beta cell autoantigens and immunocytes such as macrophages, dendritic cells, and $\mathrm{T}$ cells and their secretory products were involved in the pathogenesis of T1DM (Voorbij et al., 1989; Christianson et al., 1993; Jansen et al., 1994; Yoon and Jun, 2005). In addition, recent studies suggested that patients with T1DM have decreased numbers of endothelial progenitor cells (EPCs) and impaired functional capacity (Hill et al., 2003; Loomans et al., 2004; Tamarat et al., 2004). Folic acid (FA) is form of the water-soluble vitamin B9, which plays an important role in aiding rapid cell division and growth. Studies have shown that FA supplementation restores endothelial function in patients with coronary artery disease, hypercholesterolemia, and types 1 and 2 DM (Verhaar et al., 1998; Wilmink et al., 2000; Doshi et al., 2001; Pena et al., 2004; Title et al., 2006). However, the therapeutic potential of FA in DM remains to be fully elucidated. In addition, the effects of T1DM on EPCs are not well characterized at the molecular level.

In this study, we downloaded the gene expression profile of EPCs in T1DM patients and compared it to that of healthy controls, and then used bioinformatic-based methods to identify differential expression and its function. Furthermore, we analyzed the gene expression profiles of EPCs in FA-treated T1DM patients and compared it to that of healthy controls 
in order to investigate the outcome of FA treatment in T1DM patients. We believe that our study will improve the understanding of the molecular mechanism of T1DM and shed some light on the therapeutic studies for T1DM.

\section{MATERIAL AND METHODS}

\section{Microarray data}

Transcriptional profile of GSE17635 was downloaded from Gene Expression Omnibus, which is based on the platform of Sentrix HumanRef- 8 Expression Beadchip. This dataset was deposited by Oostrom (van Oostrom et al., 2009). EPC samples were collected from 20 patients with T1DM and 20 age- and gender-matched healthy control subjects. Patients with T1DM were then treated with FA ( $5 \mathrm{mg} /$ day) for 4 weeks, after which EPC samples were collected again. A total of 32 chips were available for further analysis, including 11 chips of EPC samples from T1DM patients, 10 chips of EPC samples from T1DM patients after FA treatment, and 11 chips of EPC samples from healthy controls.

\section{Pathway data}

Kyoto Encyclopedia of Genes and Genomes (KEGG) is a collection of online databases dealing with genomes, enzymatic pathways, and biological chemicals (Kanehisa, 2002). The PATHWAY database records networks of molecular interactions in the cells, and variants of them specific to particular organisms (http://www.genome.jp/kegg/). A total of 130 pathways, involving 2287 genes, were collected from KEGG.

\section{Data preprocessing and differential expression analysis}

We first converted the probe-level data in the CEL files into expression measures. For each sample, the expression values of all probes for a given gene were reduced to a single value by using the average expression value. We then imputed the missing data (Troyanskaya et al., 2001) and performed quartile data normalization (Fujita et al., 2006). The limma package (Diboun et al., 2006) in R Development Core Team (2011) was used to identify differentially expressed genes (DEGs) in the EPCs from patients with DM1 before and after FA treatment and to compare them with those of healthy controls. To circumvent the multi-test problem that might lead to an increase in the false-positive results, the BH method (Benjamini and Hochberg, 1995) was used to adjust the raw P values into false discovery rate (FDR). An FDR of $<$ 0.05 was used as the cut-off criterion.

\section{Pathway enrichment analysis}

The Database for Annotation, Visualization and Integrated Discovery (DAVID) provides a comprehensive set of functional annotation tools for investigators to understand the biological meaning underlying the large list of genes (Huang da et al., 2009). For functional annotation of the DEGs, we identified the overrepresented KEGG categories in pathways. A P value of $<0.05$ was used as the cut-off criterion. 


\section{RESULTS}

\section{Identification of DEGs in the EPCs from patients with T1DM before and after FA treatment}

For the dataset GSE17635, a total of 719 DEGs were identified at an FDR of 0.05, including 696 DEGs between T1DM patients and healthy controls, and 23 DEGs between T1DM patients after FA treatment and healthy controls. The number of DEGs in T1DM patients greatly decreased after treatment with FA. Thus, FA treatment can normalize gene expression to healthy control levels. The results of differential gene expression analysis are shown in Figure 1, with the Venn diagram depicting the distribution of 719 DEGs. A total of 696 genes were differentially expressed in T1DM patients compared to healthy controls, and only 23 genes were differentially expressed in T1DM patients after FA treatment compared to the healthy controls. The intersect shows that three genes (HCLS1, CECR1, and TNSRSF21) with differential expression between T1DM and healthy controls cannot be modulated by FA treatment. The remaining 20 genes may associate with side effects of FA treatment. To facilitate further analysis, we named the DEGs between T1DM patients and healthy controls (693 genes) as gene set 1, and the other two groups of DEGs were named as gene set 2 (20 genes) and gene set 3 ( 3 genes).

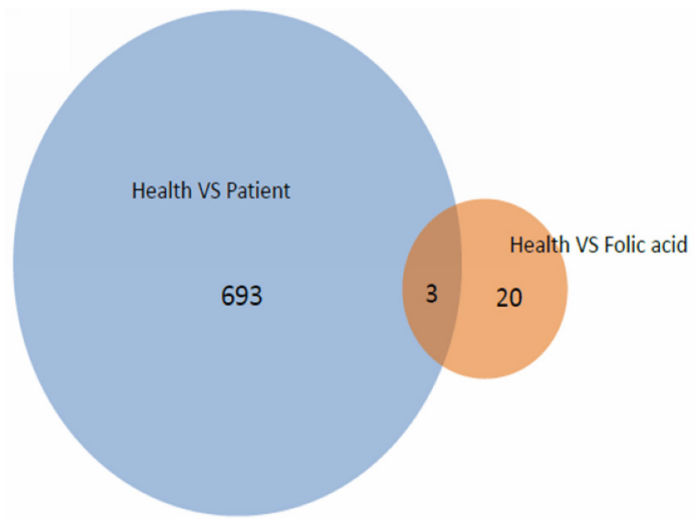

Figure 1. A Venn diagram depicting differential gene expression. The blue region represents DEGs in T1DM patients compared to healthy controls. The orange region represents DEGs in T1DM patients after FA treatment compared to healthy controls.

\section{Functional annotation of DEGs}

To investigate the effect of T1DM on EPC gene expression at the functional level, the DEGs in the EPCs of patients with T1DM before and after FA treatment compared to those of the healthy controls were input into the DAVID for pathway enrichment analysis. At a P value of 0.05 , the DEGs in gene set 1 were enriched in 3 pathways (Table 1). The most significant enrichment was the pathway of graft-versus-host disease with a P value of 0.15714 . The other significant pathways included arachidonic acid metabolism $(\mathrm{P}$ value $=0.019411)$ and antigen processing and presentation $(\mathrm{P}$ value $=0.03898)$. In fact, all significant pathways were related to immune response. The other two groups of DEGs were not enriched in any pathways. 
Table 1. The enriched pathways of differentially expressed genes between T1DM patients and healthy controls.

\begin{tabular}{llll}
\hline KEGG-ID & Term & P value & Genes \\
\hline hsa05332 & Graft-versus-host disease & 0.015714 & HLA-DPA1, HLA-B, HLA-DPB1, KIR2DL2, HLA-G, KLRC1 \\
hsa00590 & Arachidonic acid metabolism & 0.019411 & CYP2U1, GGT5, GGT6, CYP4F3, CYP4F2, PLA2G3, PLA2G5 \\
hsa04612 & Antigen processing and presentation & 0.03898 & RFX5, CREB1, HLA-DPA1, HLA-B, HLA-DPB1, KIR2DL2, HLA-G, KLRC1 \\
\hline
\end{tabular}

\section{Functional interaction network analysis of DEGs in gene sets 2 and 3}

The DEGs in gene set 1 were successfully mapped to 3 KEGG pathways, while the other two groups of DEGs were not enriched in any pathways. To further analyze these DEGs, we mapped them to the Search Tool for the Retrieval of Interacting Genes (STRING) database (Szklarczyk et al., 2011) to predict the interacting genes. A total of 2630 and 487 interacting relationships, which involved 218 and 164 genes, were identified in gene sets 2 and 3, respectively. By integrating these relationships, we constructed two interaction networks (Figures 2 and 3). Functional annotation showed that the DEGs in set 2 and their interacting genes were enriched in 11 pathways, including phosphatidylinositol signaling system, glycosphingolipid biosynthesis, and neuroactive ligandreceptor interaction (Table 2). The DEGs in set 3 and their interacting genes were enriched in 3 pathways: viral myocarditis, allograft rejection, and graft-versus-host disease (Table 3).

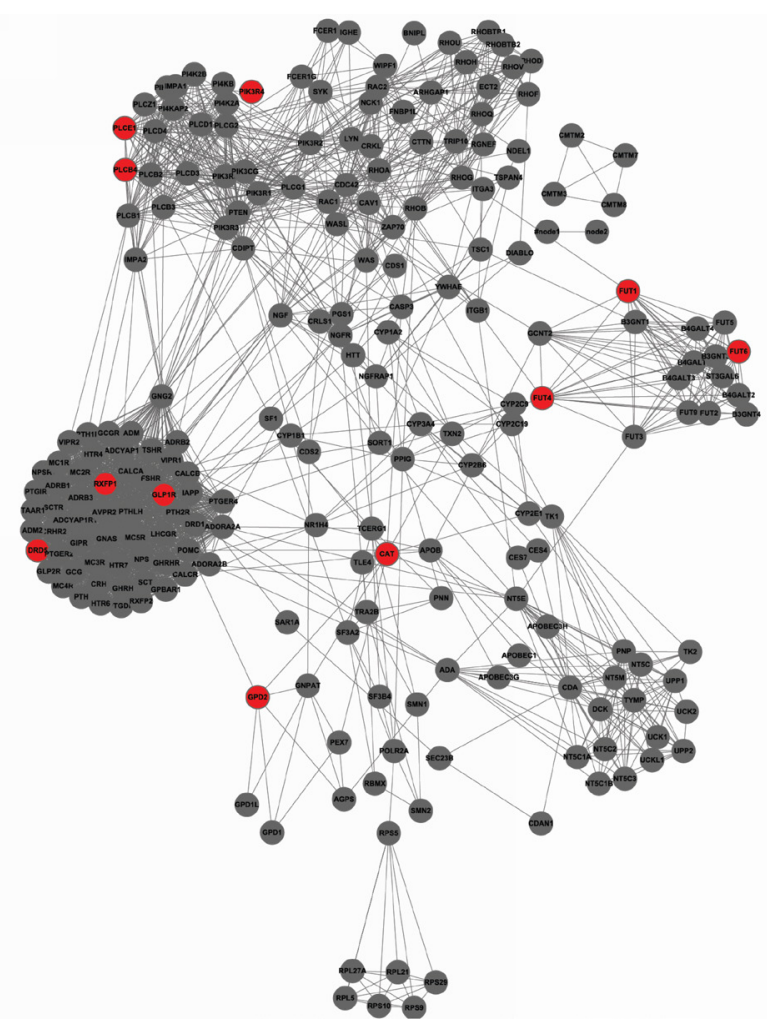

Figure 2. Interaction network constructed in gene set 2 . The red nodes represent the differentially expressed genes and the other nodes represent their interacting genes in STRING. 


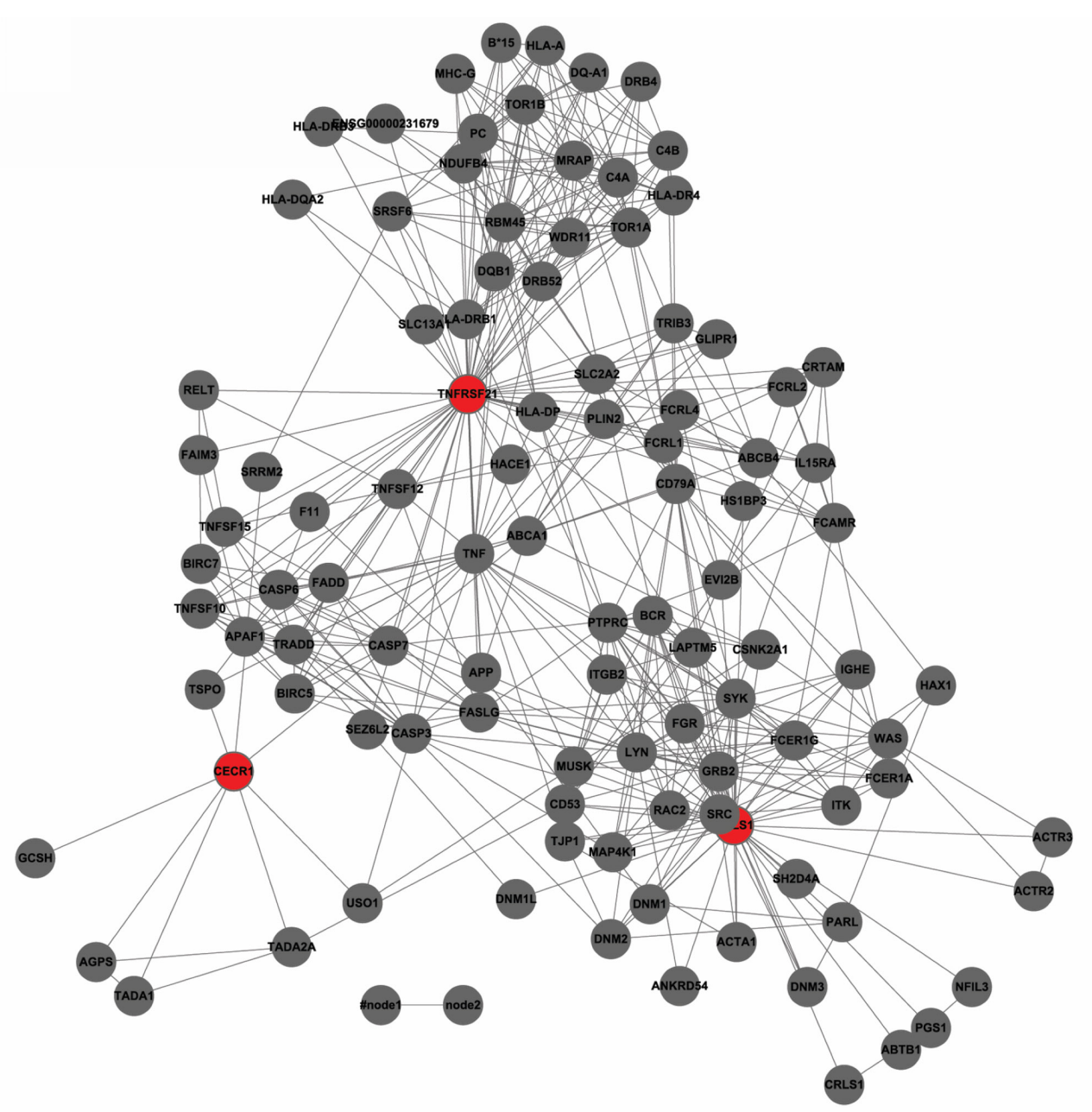

Figure 3. Interaction network constructed in gene set 3. The red nodes represent the differentially expressed genes and the other nodes represent their interacting genes in STRING.

Table 2. Enriched pathways of differentially expressed genes in gene set 2 and their interacting genes.

\begin{tabular}{lllr}
\hline KEGG-ID & Term & P & FDR \\
\hline hsa04070 & Phosphatidylinositol signaling system & $1.51 \mathrm{E}-17$ & $1.68 \mathrm{E}-14$ \\
hsa00601 & Glycosphingolipid biosynthesis & $4.50 \mathrm{E}-17$ & $5.00 \mathrm{E}-14$ \\
hsa04080 & Neuroactive ligand-receptor interaction & $1.84 \mathrm{E}-16$ & $2.44 \mathrm{E}-13$ \\
hsa00562 & Inositol phosphate metabolism & $2.64 \mathrm{E}-13$ & $2.93 \mathrm{E}-10$ \\
hsa00240 & Pyrimidine metabolism & $5.95 \mathrm{E}-10$ & $6.61 \mathrm{E}-07$ \\
hsa04020 & Calcium signaling pathway & $2.44 \mathrm{E}-08$ & $2.70 \mathrm{E}-05$ \\
hsa00983 & Drug metabolism & $6.32 \mathrm{E}-07$ & $7.01 \mathrm{E}-04$ \\
hsa04666 & Fc gamma R-mediated phagocytosis & $1.38 \mathrm{E}-06$ & 0.001529 \\
hsa04664 & Fc epsilon RI signaling pathway & $5.10 \mathrm{E}-06$ & 0.005662 \\
hsa00760 & Nicotinate and nicotinamide metabolism & $6.41 \mathrm{E}-06$ & 0.007118 \\
hsa04722 & Neurotrophin signaling pathway & $7.24 \mathrm{E}-06$ & 0.008041 \\
\hline
\end{tabular}


Table 3. The enriched pathways of differentially expressed genes in gene set 3 and their interacting genes.

\begin{tabular}{llll}
\hline KEGG-ID & Term & P & FDR \\
\hline hsa05416 & Viral myocarditis & $2.93 \mathrm{E}-05$ & 0.029327 \\
hsa05330 & Allograft rejection & $3.31 \mathrm{E}-05$ & 0.033146 \\
hsa05332 & Graft-versus-host disease & $4.57 \mathrm{E}-05$ & 0.04578 \\
\hline
\end{tabular}

\section{DISCUSSION}

In this study, we used bioinformatic methods to investigate the molecular mechanism underlying T1DM and the effects of FA on the transcriptome of EPCs from T1DM patients. The results showed that the expression of 696 genes was altered in the EPCs from T1DM patients compared to that in the EPCs from healthy controls. These genes were mainly involved in pathways associated with immune response, such as graft-versus-host disease, arachidonic acid metabolism, and antigen processing and presentation. Furthermore, we showed that FA could normalize a majority of altered gene expression profiles of EPCs from T1DM patients to resemble those of EPCs from healthy subjects, albeit with some side effects.

T1DM is related to reduced vascular repair, as indicated by impaired wound healing and reduced collateral formation in ischemia (Abaci et al., 1999). Previous studies have shown that EPC is an important regulator of these processes and that adverse metabolic stress factors in T1DM are associated with reduced EPC numbers and angiogenesis (Asahara et al., 1999; Loomans et al., 2004; Tamarat et al., 2004). To improve the impaired numerical and functional capacity of EPCs, it is necessary to identify the genes affected by T1DM. We observed an effect of T1DM on the members of the major histocompatibility complex family (HLA-DPA1, HLA-B, HLA-DPB1, and HLA-G) and killer cell immunoglobulin-like receptors (KIR2DL2 and KLRC1) involved in the pathway of graftversus-host disease. The HLA-DPA1 and DPB1 genes are the third set of classical HLA class II loci that code for the DP antigen and are associated with lower immunostimulatory capacity and level of expression (Pawelec and Buhring, 1990). Recently, Varney et al. (2010) suggested these two genes contributed to the risk associated with T1DM. The major histocompatibility complex class I genes (HLA-B and HLA-G) were also found to contribute to the etiology of T1DM (Abediankenari et al., 2007; Nejentsev et al., 2007). Killer cell immunoglobulin-like receptors on chromosome 19q13.4 regulate the function of not only human natural killer cells but also T cells. Ramos-Lopez suggested a potential role of the KIR2DL2-rs 2756923 polymorphism in T1DM in the German and Belgian populations (Ramos-Lopez et al., 2009).

To further explore the molecular mechanism of T1DM, we analyzed the most significant dysfunctional pathway of graft-versus-host disease in KEGG (Figure 4). Graft-versushost disease is a lethal complication of allogeneic hematopoietic stem cell transplantation, wherein the immunocompetent donor $\mathrm{T}$ cells attack the genetically disparate host cells. The pathophysiology of graft-versus-host disease can be summarized in a three-step process, and nitric oxide (NO) is produced by activated macrophages during step 2. Hyperglycemia increases the production of superoxide $\left(\mathrm{O}_{2}^{-}\right)$and reduces the bioavailability of NO resulting in the development of endothelial dysfunction in DM patients (Dimmeler et al., 2002; Creager et al., 2003). Therefore, we suggested that the production of NO during step 2 of the graft-versushost disease plays a role in EPC dysfunction in patients with T1DM. 


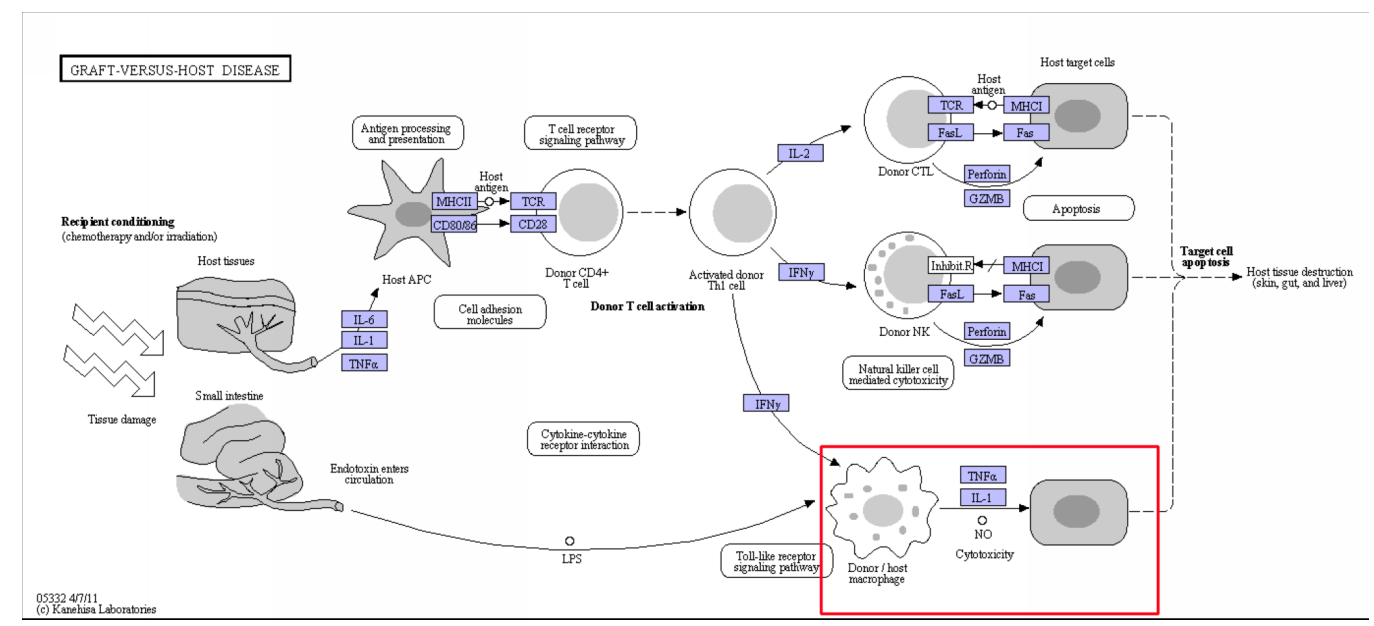

Figure 4. KEGG map of graft-versus-host disease. The red box indicated the production of NO during step 2 of GVHD.

Previous studies have shown that FA exerts protective anti-oxidant effects and improves endothelial function in patients with cardiovascular diseases (Doshi et al., 2001; Pena et al., 2004; Antoniades et al., 2006). In this study, we assessed the effect of FA treatment on T1DM by analyzing the gene expression profile of EPCs from T1DM patients treated with FA and comparing it to that from healthy controls. Indeed, FA normalized a majority of changes in gene expression induced by T1DM (99.6\%), suggesting the therapeutic potential of FA in the treatment of T1DM. However, we also found 20 DEGs between T1DM patients and healthy controls. These differential expressions were induced by FA treatment, and they might be associated with the side effects of FA treatment. Functional interaction network analysis showed that FA treatment might cause dysregulation of metabolism, such as in case of inositol phosphate metabolism and pyrimidine metabolism. Therefore, we suggested that focused efforts should be invested to ensure that the dose of FA falls within the pharmacological range, especially in case of diabetic pregnancy (Capel and Corcoy, 2007). This result is inconsistent with that of the original study, which suggested that the gene expression profiles of EPCs from healthy controls and T1DM patients after FA treatment were very similar and that pairwise comparison showed no DEGs at an FDR of 0.05 (van Oostrom et al., 2009). We hypothesized that these differences may be due to the different method used for the analysis of microarray data.

In conclusion, our study employed bioinformatics methods to elucidate the molecular mechanism underlying T1DM and the effects of FA treatment on the transcriptome of EPCs from patients with T1DM. We observed a remarkable effect of FA treatment with respect to normalization of the altered gene expression induced by T1DM, and a new differential gene expression pattern that may be associated with the potential side effects of FA treatment.

\section{REFERENCES}

Abaci A, Oguzhan A, Kahraman S, Eryol NK, et al. (1999). Effect of diabetes mellitus on formation of coronary collateral vessels. Circulation 99: 2239-2242. 
Abediankenari S, Eslami MB, Sarrafnejad A, Mohseni M, et al. (2007). Dendritic cells bearing HLA-G inhibit T-Cell activation in type 1 diabetes. Iran J. Allergy Asthma Immunol. 6: 1-7.

Antoniades C, Shirodaria C, Warrick N, Cai S, et al. (2006). 5-methyltetrahydrofolate rapidly improves endothelial function and decreases superoxide production in human vessels: effects on vascular tetrahydrobiopterin availability and endothelial nitric oxide synthase coupling. Circulation 114: 1193-1201.

Asahara T, Masuda H, Takahashi T, Kalka C, et al. (1999). Bone marrow origin of endothelial progenitor cells responsible for postnatal vasculogenesis in physiological and pathological neovascularization. Circ. Res. 85: 221-228.

Atkinson MA and Maclaren NK (1994). The pathogenesis of insulin-dependent diabetes mellitus. N. Engl. J. Med. 331: 1428-1436.

Benjamini Y and Hochberg Y (1995). Controlling the false discovery rate: a practical and powerful approach to multiple testing. J. R. Stat. Soc. Series B 57: 289-300.

Capel I and Corcoy R (2007). What dose of folic acid should be used for pregnant diabetic women? Diabetes Care 30: e63.

Christianson SW, Shultz LD and Leiter EH (1993). Adoptive transfer of diabetes into immunodeficient NOD-scid/scid mice. Relative contributions of CD4+ and CD8+ T-cells from diabetic versus prediabetic NOD.NON-Thy-1a donors. Diabetes 42: 44-55.

Creager MA, Luscher TF, Cosentino F and Beckman JA (2003). Diabetes and vascular disease: pathophysiology, clinical consequences, and medical therapy: Part I. Circulation 108: 1527-1532.

Daneman D (2006). Type 1 diabetes. Lancet 367: 847-858.

Diboun I, Wernisch L, Orengo CA and Koltzenburg M (2006). Microarray analysis after RNA amplification can detect pronounced differences in gene expression using limma. BMC Genomics 7: 252.

Dimmeler S, Haendeler J and Zeiher AM (2002). Regulation of endothelial cell apoptosis in atherothrombosis. Curr. Opin. Lipidol. 13: 531-536.

Doshi SN, McDowell IF, Moat SJ, Lang D, et al. (2001). Folate improves endothelial function in coronary artery disease: an effect mediated by reduction of intracellular superoxide? Arterioscler. Thromb. Vasc. Biol. 21: 1196-1202.

Fujita A, Sato JR, Rodrigues LO, Ferreira CE, et al. (2006). Evaluating different methods of microarray data normalization. BMC Bioinformatics 7: 469.

Hill JM, Zalos G, Halcox JP, Schenke WH, et al. (2003). Circulating endothelial progenitor cells, vascular function, and cardiovascular risk. N. Engl. J. Med. 348: 593-600.

Huang dW, Sherman BT and Lempicki RA (2009). Systematic and integrative analysis of large gene lists using DAVID bioinformatics resources. Nat. Protoc. 4: 44-57.

Jansen A, Homo-Delarche F, Hooijkaas H, Leenen PJ, et al. (1994). Immunohistochemical characterization of monocytesmacrophages and dendritic cells involved in the initiation of the insulitis and beta-cell destruction in NOD mice. Diabetes 43: 667-675.

Kanehisa M (2002). The KEGG database. Novartis Found. Symp. 247: 91-101.

Loomans CJ, de Koning EJ, Staal FJ, Rookmaaker MB, et al. (2004). Endothelial progenitor cell dysfunction: a novel concept in the pathogenesis of vascular complications of type 1 diabetes. Diabetes 53: 195-199.

Nathan DM, Lachin J, Cleary P, Orchard T, et al. (2003). Intensive diabetes therapy and carotid intima-media thickness in type 1 diabetes mellitus. N. Engl. J. Med. 348: 2294-2303.

Nejentsev S, Howson JM, Walker NM, Szeszko J, et al. (2007). Localization of type 1 diabetes susceptibility to the MHC class I genes HLA-B and HLA-A. Nature 450: 887-892.

Pawelec G and Buhring HJ (1990). Expression of MHC class II epitopes on human T lymphocyte clones. Cell Immunol. 127: 520-526.

Pena AS, Wiltshire E, Gent R, Hirte C, et al. (2004). Folic acid improves endothelial function in children and adolescents with type 1 diabetes. J. Pediatr. 144: 500-504.

R Development Core Team (2011). R: A Language and Environment for Statistical Computing. R Foundation for Statistical Computing, Vienna.

Ramos-Lopez E, Scholten F, Aminkeng F, Wild C, et al. (2009). Association of KIR2DL2 polymorphism rs2756923 with type 1 diabetes and preliminary evidence for lack of inhibition through HLA-C1 ligand binding. Tissue Antigens 73: 599-603.

Schranz DB and Lernmark A (1998). Immunology in diabetes: an update. Diabetes Metab. Rev. 14: 3-29.

Sheetz MJ and King GL (2002). Molecular understanding of hyperglycemia's adverse effects for diabetic complications. JAMA 288: 2579-2588.

Szklarczyk D, Franceschini A, Kuhn M, Simonovic M, et al. (2011). The STRING database in 2011: functional interaction networks of proteins, globally integrated and scored. Nucleic Acids Res. 39: D561-D568.

Tamarat R, Silvestre JS, Le Ricousse-Roussanne S, Barateau V, et al. (2004). Impairment in ischemia-induced neovascularization in diabetes: bone marrow mononuclear cell dysfunction and therapeutic potential of placenta 
growth factor treatment. Am. J. Pathol. 164: 457-466.

Title LM, Ur E, Giddens K, McQueen MJ, et al. (2006). Folic acid improves endothelial dysfunction in type 2 diabetes-an effect independent of homocysteine-lowering. Vasc. Med. 11: 101-109.

Troyanskaya O, Cantor M, Sherlock G, Brown P, et al. (2001). Missing value estimation methods for DNA microarrays. Bioinformatics 17: 520-525.

van Oostrom O, de Kleijn DP, Fledderus JO, Pescatori M, et al. (2009). Folic acid supplementation normalizes the endothelial progenitor cell transcriptome of patients with type 1 diabetes: a case-control pilot study. Cardiovasc. Diabetol. 8: 47.

Varney MD, Valdes AM, Carlson JA, Noble JA, et al. (2010). HLA DPA1, DPB1 alleles and haplotypes contribute to the risk associated with type 1 diabetes: analysis of the type 1 diabetes genetics consortium families. Diabetes 59: 2055-2062.

Verhaar MC, Wever RM, Kastelein JJ, van DT, et al. (1998). 5-methyltetrahydrofolate, the active form of folic acid, restores endothelial function in familial hypercholesterolemia. Circulation 97: 237-241.

Voorbij HA, Jeucken PH, Kabel PJ, De HM, et al. (1989). Dendritic cells and scavenger macrophages in pancreatic islets of prediabetic BB rats. Diabetes 38: 1623-1629.

Williams SB, Cusco JA, Roddy MA, Johnstone MT, et al. (1996). Impaired nitric oxide-mediated vasodilation in patients with non-insulin-dependent diabetes mellitus. J. Am. Coll. Cardiol. 27: 567-574.

Wilmink HW, Stroes ES, Erkelens WD, Gerritsen WB, et al. (2000). Influence of folic acid on postprandial endothelial dysfunction. Arterioscler. Thromb. Vasc. Biol. 20: 185-188.

Yoon JW and Jun HS (2005). Autoimmune destruction of pancreatic beta cells. Am. J. Ther. 12: 580-591. 